\title{
Disfunción ileostómica en pacientes sometidos a resección anterior baja por un cáncer del recto luego de neoadyuvancia
}

\author{
Guillermo Bannura C. ${ }^{1}$, Macarena Fernández A. ${ }^{1}$, Benjamín Fernández M. ${ }^{1}$, \\ Alejandro Barrera E. ${ }^{1}$, Carlos Melo L. ${ }^{1}$, Felipe Illanes F. ${ }^{1}$, \\ Cristián Gallardo V. ${ }^{1}$ y Nelson Muñoz P. ${ }^{1}$
}

'Servicio y Departamento de Cirugía Hospital Clínico San Borja Arriarán. Campus Centro, Facultad de Medicina Universidad de Chile. Santiago, Chile.

Recibido 2020-06-05 y aceptado 2020-07-15

Correspondencia a: Dr. Guillermo Bannura C. gbannura@gtdmail.com
Ileostomic dysfunction in patients undergoing low anterior resection for rectal cancer after neoadjuvant treatment

Background: Diverting loop ileostomy (LI) is commonly performed to protect a distal anastomosis after a low anterior resection. Aim: To investigate the frecuency and clinical features of ileostomic dysfunction (ID) and, eventually, to define a profile of patients at risk of this complicaction. Materials and Method: 103 consecutive patients operated on for rectal cancer were included. ID is defined when the maxime output was more than $1,5 \mathrm{lt} /$ day for three or more consecutive days with biochemical disturbances, associated to abdominal distension and the inability to tolerate oral feeding without postoperative severe complication. Patients with ID were compared with noID group. Results: ID developed in 15 patients, lasting between 12 and 70 days (50\% for more then 30 days), the readmission rate was $27 \%$ without reoperation in this group. Except for inhospital time and readmission rate, no other difference between both groups were founded. Discussion: ID is a kind of paralitic ileus of variable intensity with paradox high output ileostomy leading to depletion of water and electrolyte imbalance. ID requires reposition of high volumen of fluids and electrolytes and sometimes parenteral nutrition with full recovery in variable periods without invasive treatment. In this study it was not possible to define a patient profile at risk or predictive factores of this complication.

Key words: loop ileostomy; complications; low anterior resection; rectal cancer.

\section{Resumen}

Introducción: La resección anterior baja protegida con una ileostomía en asa (IA) luego de neoadyuvancia es el tratamiento estándar del cáncer del recto bajo localmente avanzado. Objetivos: Investigar la incidencia, características clínicas de la disfunción ileostómica (DI) en estos pacientes y, eventualmente, definir un perfil de riesgo. Materiales y Método: Se analizan 103 pacientes consecutivos. La DI se define como la eliminación por la ileostomía de más de 1,5 litros por día durante 3 o más días consecutivos asociado a distensión y dolor abdominal con intolerancia a la alimentación oral en ausencia de una complicación intraabdominal Clavien-Dindo grado III o mayor. Se comparan el grupo con DI del resto (no DI). Resultados: La DI se presentó en el 14,5\% de los casos, se resolvió entre 12 y 70 días (en el $50 \%$ superó los 30 días), la tasa de reingreso fue $27 \%$ y no hubo reoperaciones en este grupo. No hubo diferencias estadísticamente significativas entre ambos grupos salvo en el tiempo de hospitalizaión y la tasa de reingresos. Discusión: La DI corresponde a un tipo de íleo posoperatorio de gravedad y duración variable que paradojalmente se asocia con la eliminación de altos volúmenes de contenido intestinal por la IA, requiere aporte vigoroso de volumen y electrolitos y en los casos más graves apoyo con nutrición parenteral. El cuadro revierte en plazos variables con manejo conservador. En este estudio no se ha logrado definir un perfil del paciente en riesgo de sufrir esta complicación o factores predictivos de ella.

Palabras clave: ileostomía en asa; complicaciones de la ileostomía; resección anterior baja; cáncer del recto. 


\section{Introducción}

El tratamiento estándar del cáncer del recto bajo en la actualidad se basa en la resección radical del segmento comprometido luego de algún esquema de neoadyuvancia ${ }^{1}$. En los procedimientos con conservación esfinteriana se aconseja proteger la anastomosis colorrectal baja mediante una ileostomía en asa, con el fin de prevenir las graves complicaciones sépticas asociadas a la falla anastomótica, especialmente en pacientes irradiados con anastomosis muy bajas $^{2}$.

La ileostomía en asa (IA) efectuada luego de una resección anterior baja o ultrabaja tiene morbilidad propia, destacando el desbalance hidroelectrolítico por pérdidas excesivas, una mayor frecuencia de íleo paralítico posoperatorio y un aumento en el tiempo de hospitalización necesario para estabilizar al paciente ${ }^{3}$.

No existe en la literatura especializada una definición universal sobre algunas complicaciones asociadas a la IA, las cuales se imbrican y se confunden como la ileostomía de alto débito (HOI, por sus siglas en inglés), la obstrucción de salida (stoma outlet obstruction) y el íleo paralítico prolongado, las cuales ocurren en el periodo posoperatorio inmediato $^{2,3}$.

En un estudio previo, definimos la disfunción ileostómica (DI) como una forma de íleo posoperatorio caracterizado por la falta de eliminación de gases y contenido líquido por la IA durante 3 a 5 días, acompañado de distensión abdominal y mala tolerancia a la realimentación oral progresiva. Luego se produce una descarga de grandes volúmenes de contenido intestinal de color verde botella (sobre 1,5 litros diarios durante varios días, durante el cual el paciente persiste distendido, no tolera la realimentación y requiere de una vigorosa reposición de volumen y electrolitos y, eventualmente, apoyo con nutrición parenteral. Finalmente, el cuadro progresa espontáneamente hacia la mejoría en tiempos variables, disminuyendo los débitos de la ileostomía, ocurre un cambio paulatino de la coloración y consistencia de las heces, se aprecia la presencia de gases en la bolsa y se observa la tolerancia progresiva a la alimentación oral ${ }^{4}$.

El objetivo de este estudio es investigar la incidencia de la DI en pacientes irradiados sometidos a una resección anterior baja o ultrabaja por un cáncer del recto, analizar las características clínicas de la DI y, eventualmente, definir el perfil del paciente en riesgo de presentar esta complicación.

\section{Materiales y Método}

Estudio retrospectivo en el cual se incluyen todos los pacientes intervenidos por un cáncer de recto bajo con intención curativa luego de radioquimioterapia preoperatoria con conservación esfinteriana cuya anastomosis quedó protegida con una IA. Se define la DI como la eliminación en el posoperatorio inmediato de un débito mayor de 1,5 litros por más de 3 días consecutivos, asociado a la incapacidad de tolerar la realimentación oral y al desbalance hidroelectrolítico secundario, en ausencia de una complicación grave y con una resolución espontánea a la mejoría. No se incluyen los pacientes que reingresan por deshidratación y/o falla renal en el posoperatorio mediato debido a las pérdidas excesivas de la IA que no son compensadas por una realimentación oral tolerada en forma satisfactoria. Se excluyen los pacientes estadio IV sometidos a una resección radical y las ileostomías en el contexto de una exenteración pélvica total.

Para identificar un potencial perfil del paciente susceptible de sufrir esta complicación se compara el grupo que presentó una DI con el grupo que no presentó esta complicación (no DI) según características demográficas, comorbilidades, localización del tumor, antígeno carcinoembrionario y otras variables preoperatorias potencialmente asociadas con la DI. Se utilizó el índice de comorbilidad de Charlson $^{5}$ para evaluar el riesgo quirúrgico (0-1 punto: ausencia de morbilidad; 2 puntos: morbilidad baja; 3 o más puntos: morbilidad alta. Según este score la enfermedad neoplásica per se da 2 puntos). $\mathrm{La}$ severidad de las complicaciones posoperatorias se graduó de acuerdo con la clasificación de Clavien-Dindo ${ }^{6}$.

El manejo posoperatorio fue estandarizado en todos los pacientes de acuerdo con un protocolo ERAS parcial, que incluye manejo del dolor mediante epidural + analgésicos endovenosos, levantada precoz y realimentación líquida progresiva desde el día 1 de la intervención hasta alcanzar el régimen blando sin residuos rico en pectina. En los pacientes con buena tolerancia alimentaria y débitos altos se indicó loperamida en dosis crecientes hasta un máximo de $16 \mathrm{mg}$ /día. Se confeccionó una tabla de colores para definir el contenido recolectado en la bolsa como un indicador de la función intestinal y la eventual asociación con la recuperación del íleo posoperatorio (Figura 1). El alta se autorizó cuando los débitos eran alrededor de $700 \mathrm{ml} /$ día con deposiciones cremosas de color café (tipo 4). 


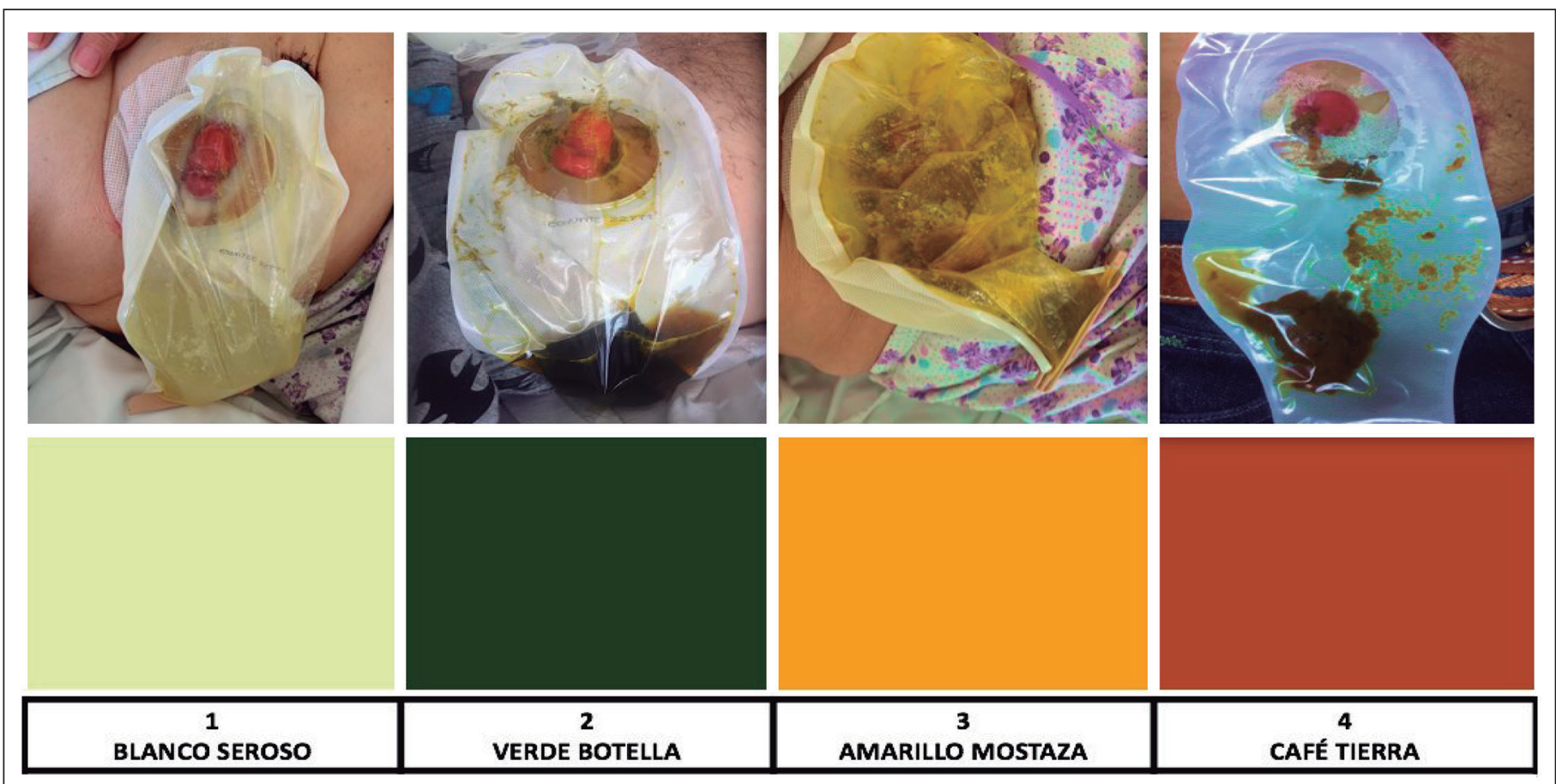

Figura 1. Cambios de color y consistencia de una ileostomía en el posoperatorio.

Para el análisis estadístico se empleó el test exacto de Fischer para variables categóricas y el test de Wilkonson para comparar variables continuas.

\section{Resultados}

En el estudio se incluyen 103 pacientes portadores de un cáncer de recto bajo sometidos a neoadyuvancia seguida de una resección radical con intención curativa con una anastomosis protegida por una ileostomía en asa en forma consecutiva en un periodo de 15 años, el 32\% de ellas por vía laparoscópica. Las características demográficas y las variables consideradas en ambos grupos se aprecian en la Tabla 1, sin diferencias significativas salvo en el CEA preoperatorio promedio que es mayor en el grupo No DI. Los resultados de la cirugía y el estadio patológico definitivo se muestran en la Tabla 2. Sólo la mayor incidencia de los reingresos

Tabla 1. Características demográficas según DI

\begin{tabular}{|lccc|}
\hline Variable & $\begin{array}{c}\text { No DI } \\
\mathbf{n}=\mathbf{8 8}\end{array}$ & $\begin{array}{c}\text { DI } \\
\mathbf{n = 1 5}\end{array}$ & Valor p \\
\hline Edad promedio (extremos) & $62(28-82)$ & $65(46-82)$ & 0,353 \\
Género femenino & $56,8 \%$ & $46,7 \%$ & 0,465 \\
\hline I. Charlson mediana (extremos) & $4(2-7)$ & $4(2-9)$ & 0,091 \\
Hto preop. promedio & $47,7 \%$ & $38,8 \%$ & 0,434 \\
Albúmina preop. promedio (mg/dl) & 4,06 & 4,12 & 0,603 \\
CEA preop. promedio (ng/ml) & 10,88 & 3,71 & 0,040 \\
Proporción N/L preop. & 2,5 & 2,7 & 0,570 \\
Localización RM/RI & $72 / 16$ & $13 / 2$ & 0,648 \\
\hline Altura de la anastomosis $(\mathrm{cm})$ & 5,40 & 5,13 & 0,650 \\
\hline
\end{tabular}

Índice de Charlson. Hto: hematocrito. CEA: antígeno carcinoembrionario. N/L: proporción neutrófilos/linfocitos preoperatorios. RM: recto medio. RI: recto inferior. 
ARTíCULO ORIGINAL

y el mayor tiempo de hospitalización del grupo DI alcanzó significación estadística entre ambos grupos.

De acuerdo con la definición utilizada, la tasa de DI en esta serie es de $14,5 \%$ y en 14 de los 15 pacientes los débitos fueron superiores a 2 litros en $24 \mathrm{~h}$ por 3 o más días consecutivos, con extremos de 5,5 litros (Figura 2). No hubo reoperaciones y dos pacientes fueron tratados con antibióticos por la presencia de una dehiscencia anastomótica con escasa repercusión sistémica, evolución satisfactoria y alta a los 12 y 14 días, respectivamente (caso ${ }^{\circ}$ 6: una colección pélvica a la tomografía computada y caso $n^{\circ}$ 9: salida de secreción turbia por el drenaje). En el contexto de la DI, 14 pacientes presentaron vómitos, 13 requirieron de una sonda nasogástrica (SNG) y 8 recibieron nutrición parenteral por una vía central. Los débitos variables de la SNG fluctuaron entre $50 \mathrm{ml}$ de líquido bilioso de retención hasta 3.000
Tabla 2. Resultados y estadio patológico definitivo según DI

\begin{tabular}{|lccc|}
\hline Variable & $\begin{array}{c}\text { No DI } \\
\mathbf{n = 8 8}\end{array}$ & $\begin{array}{c}\text { DI } \\
\mathbf{n = 1 5}\end{array}$ & Valor $\mathbf{p}$ \\
\hline Fístula-DA-colección pélvica, n (\%) & $16(18)$ & $2(13)^{*}$ & 0,178 \\
\hline Reingresos, n (\%) & $7(7,9)$ & $4(27)$ & 0,002 \\
\hline Reoperaciones, n (\%) & $6(6,8)$ & 0 & 0,297 \\
\hline Estadio patológico I & $28(32)$ & $6(40)$ & 0,533 \\
\hline IIA & $31(35)$ & $7(47)$ & 0,396 \\
IIB & $6(7)$ & 0 & 0,297 \\
IIIA & $4(4,5)$ & 0 & 0,400 \\
IIIB & $13(15)$ & $2(13)$ & 0,884 \\
\hline IIIC & $6(7)$ & 0 & 0,297 \\
\hline Hospitalización, promedio días (extremos) & $14(4-77)$ & $21(12-41)$ & 0,018 \\
\hline
\end{tabular}

DA: dehiscencia anastomosis. *Clavien-Dindo II.

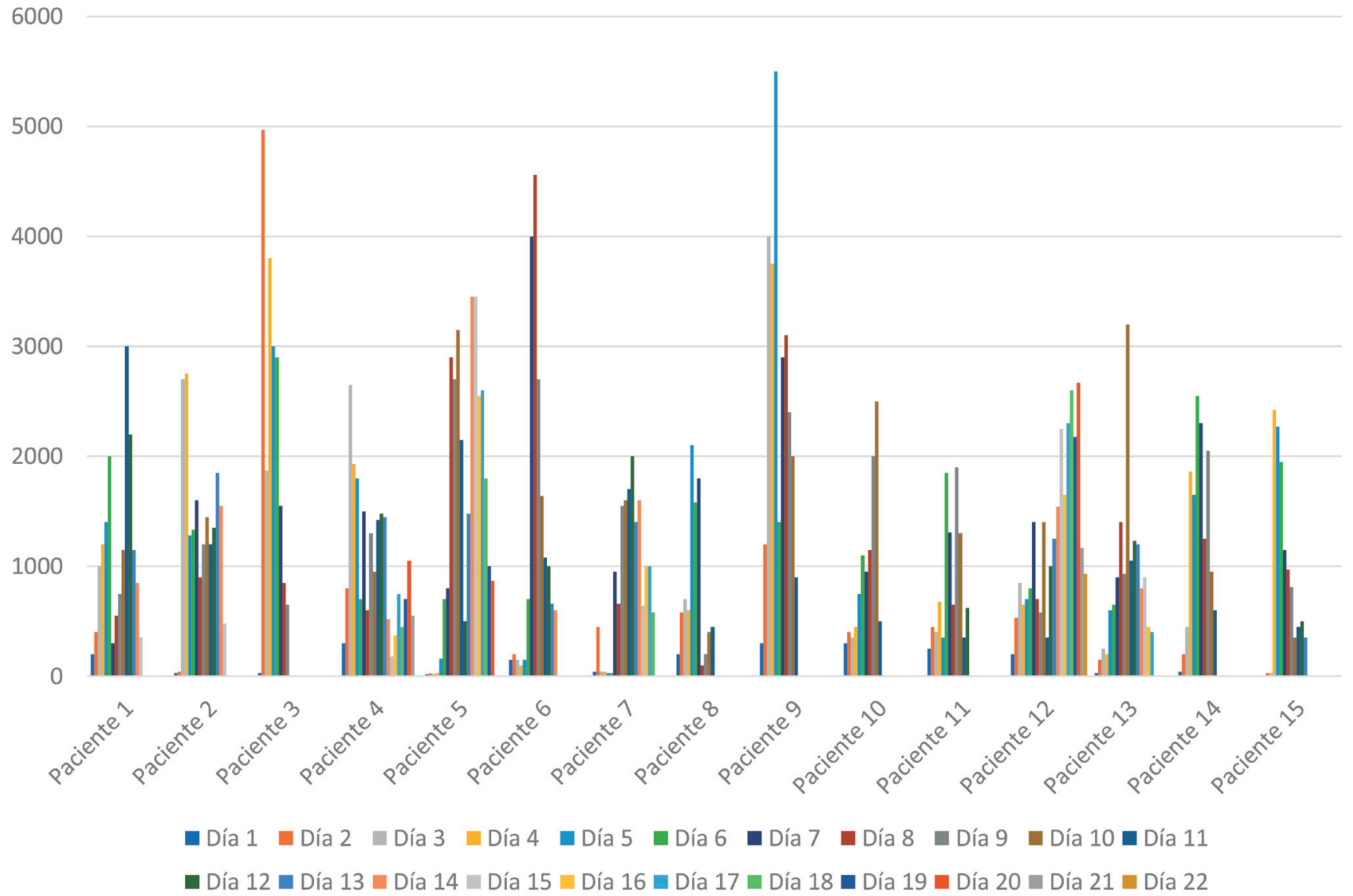

Figura 2. Débitos de la ileostomía hasta tolerancia al régimen o alta (3 semanas)* . No considera los reingresos. 


\begin{tabular}{|c|c|c|c|c|c|c|c|c|c|c|c|c|c|c|c|}
\hline $\begin{array}{l}\text { Paciente/ } \\
\text { día postop. }\end{array}$ & 2 & 6 & 10 & 14 & 18 & 22 & 26 & 30 & 34 & 38 & 42 & 46 & 54 & total días & $\begin{array}{c}\text { cierre } \\
\text { ileostomía }\end{array}$ \\
\hline 1 & 200 & 2000 & 2800 & 300 & 1150 & 550 & 350 & alta & & reingreso & 2500 & 1150 & 900 & 65 & sí \\
\hline 2 & 30 & 2750 & 1600 & 1200 & 750 & alta & reingreso & 1000 & 1580 & 1850 & 920 & 580 & alta & 48 & sí \\
\hline 3 & 1550 & 4970 & 650 & alta & & & & & & & & & & 12 & sí \\
\hline 4 & 800 & 2650 & 1800 & 2000 & 1450 & 180 & 550 & 700 & 650 & 1050 & 550 & alta & & 44 & sí \\
\hline 5 & 25 & 30 & 800 & 2900 & 3150 & 1480 & 3450 & 1800 & 2500 & 400 & 1800 & 650 & alta & 53 & sí \\
\hline 6 & 200 & 4000 & 2700 & 650 & alta & & & & & & & & & 14 & sí \\
\hline 7 & 450 & 20 & 730 & 920 & 1560 & 1000 & 1000 & 580 & alta & & & & & 35 & no \\
\hline 8 & 200 & 600 & 2100 & 210 & 400 & alta & & & & & & & & 22 & sí \\
\hline 9 & 300 & 4000 & 5500 & alta & & & & & & & & & & 14 & sí \\
\hline 10 & 350 & 780 & 2500 & alta & & & & & & & & & & 12 & sí \\
\hline 11 & 250 & 400 & 1850 & alta & & & & & & & & & & 13 & sí \\
\hline 12 & 530 & 800 & 1400 & 2600 & 1450 & alta & reingreso & 170 & 350 & 700 & 1150 & alta & & 45 & sí \\
\hline 13 & 200 & 600 & 3200 & 1230 & 400 & alta & reingreso & 350 & alta & reingreso & 1230 & 900 & cierre & 70 & sí \\
\hline 14 & 450 & 2050 & 950 & alta & & & & & & & & & & 12 & sí \\
\hline 15 & 30 & 2420 & 1970 & alta & & & & & & & & & & 14 & sí \\
\hline
\end{tabular}

Figura 3. Pacientes con disfunción ileostómica: evolución esquemática en el tiempo y reingresos*. *Por razones de espacio se registran los débitos cada 4 días. Ello significa que algunos pacientes tuvieron débitos muy altos no anotados y normalizados al alta.

$\mathrm{ml}$ en $24 \mathrm{~h}$ por periodos variables. La interrupción de la realimentación y/o vómitos ocurrió en $4,3,2$, 3,1 y 1 pacientes en los días $2,3,45,6$, y 7 días del posoperatorio, respectivamente (en 12 de los 14 casos ello ocurrió entre el $2^{\circ}$ y el $5^{\circ}$ día). El único paciente sin vómitos y que toleró un aporte oral mínimo presentó débitos por la ileostomía de 3.550 , $4.950,1.850,3.800$ y $2.900 \mathrm{ml}$ diarios en cinco días consecutivos a partir del día $3^{\circ}$ para estabilizarse posteriormente en $650 \mathrm{ml}$ al día, con una hospitalización de 12 días y sin reingreso (Figura 3). En el grupo DI hubo 4 reingresos debido a deshidratación y falla renal secundaria debido a débitos altos no compensados por una ingesta bien tolerada en tres pacientes y en 1 caso por falta de ingesta con débitos normales en el contexto de una depresión grave, por lo cual se reconstituyó en el segundo reingreso. En globo, el cuadro se resolvió entre 12 y 70 días y en la mitad de los casos la duración de la DI superó los 30 días.

La tasa de reoperaciones en el grupo no DI alcanzó al 6,8\% (6/88), debido a una dehiscencia anastomosis ( 4 casos que se transforman en una operación tipo Hartmann), una obstrucción por bridas (enterolisis) y una perforación en la base de la ileostomía (reileostomía). Ninguno de estos pacientes no DI presentó débitos elevados de la ileostomía.

\section{Discusión}

El concepto de DI fue utilizado en el pasado para hacer referencia a la obstrucción de salida de una ileostomía dejada "en maduración", secundaria a la serositis constrictiva que ocurría con alta frecuencia al dejar la serosa expuesta. Luego de los trabajos de Brooke $^{7}$ (ileostomía terminal) y de Turnbull ${ }^{8}$ (IA) se incorporan los conceptos de ostomía protruida, evertida y madurada que es la forma habitual y correcta de confección de una ileostomía, con lo cual prácticamente desapareció esta complicación. En la actualidad, se describe como complicación la ileostomía de alto débito (high-output ileostomy, HOI) cuyas características clínicas no están detalladas en la literatura especializada. Algunos estudios definen esta complicación cuando los débitos superan los 1,5 litros diarios y otros por encima de 2 litros al día, por un periodo de 3 o más días consecutivos con alteración del equilibrio hidroelectrolítico en el periodo posoperatorio inmediato ${ }^{9-12}$. La incidencia de HOI precoz es $24 \%$ promedio, aunque las series son muy heterogéneas e incluyen pacientes intervenidos por enfermedades inflamatorias, ileostomías de protección de un reservorio ileal y pacientes neoplásicos no sometidos a neoadyuvancia. Esta heterogeneidad de las series también puede explicar 
los resultados tan disímiles en cuanto a los factores predictivos de la complicación. En un estudio mejicano sobre 102 pacientes sometidos a cirugía colorrectal identifican a la colitis ulcerosa y un débito mayor a 1 litro de la ileostomía al alta son factores predictivos de HOI que ocurrió en el $24 \%$ de los casos $^{9}$. Fujino ${ }^{10}$ analiza 60 pacientes intervenidos por cáncer del recto mediante un modelo predictivo que concluye que los jóvenes, el género masculino y el recuento de glóbulos blancos elevados en el día $1^{\circ}$ del posoperatorio son predictivos de un HOI. Take$\mathrm{da}^{11}$, en un estudio de 151 pacientes intervenidos por cáncer señala que la diabetes y la pan-proctocolectomía son factores predictivos de esta complicación. Hiramoto $^{13}$, estudia 45 pacientes intervenidos por cáncer, de los cuales 22 presentaron un HOI. No hubo diferencias entre ambos grupos en la PCR, albúmina hemoglobina y comorbilidades, excepto que la relación neutrófilos/linfocitos preoperatorios fue significativamente mayor en el grupo HOI. Aparentemente este cuadro de HOI primario, es decir, en ausencia de una complicación posoperatoria ${ }^{14}$, no presenta las características clínicas de la DI y se trata de un desbalance entre los ingresos y las pérdidas en el contexto de un paciente que tolera la realimentación progresiva, pero que es insuficiente para reponerlas.

En la literatura se menciona la obstrucción de salida como una complicación precoz de la IA que se caracteriza por dolor y distensión abdominal, la falta de eliminación de contenido intestinal y/o gases, junto a la dificultad de tolerar la realimentación oral, lo que se traduce en la tomografía por una dilatación de las asas intestinales hasta el nivel de la entrada de la IA en la pared abdominal. A diferencia de la DI, este cuadro se resuelve completamente con la introducción de una sonda a través de la ileostomía durante unos días y la etiología probablemente sea edema o torsión parcial del asa exteriorizada ${ }^{15,16}$.

Algunos elementos clínicos que definen la DI corresponden a un íleo paralítico prolongado que incluye la distensión y el dolor abdominal no cólico, la ausencia de eliminación de gases/heces, acompañado de la intolerancia a la realimentación oral. Paradojalmente en la DI este cuadro clínico se acompaña de la eliminación de grandes volúmenes de contenido intestinal de color verde botella, característico de una alteración en la capacidad de absorción del intestino delgado. El cambio progresivo de color hacia el amarillo oro y el café tierra coincide con un aumento de la consistencia de las deposiciones y la reducción paulatina de los débitos, el aplanamiento del abdomen y la tolerancia al aporte oral progresivo.
En esta serie, específica de pacientes irradiados portadores de una ileostomía en asa de protección de una anastomosis colorrectal baja, la incidencia de DI es de 14,5\%, sin lograr definir un perfil del paciente susceptible ni encontrar factores de riesgo que permitan una mejor estrategia y manejo de la complicación. Hemos confirmado la incidencia, las características clínicas del cuadro, así como el manejo estandarizado y el impacto en hospitalización y reingresos en una cohorte de 103 pacientes. El periodo de aparición de la complicación fluctúa entre el $2^{\circ}$ y el $7^{\circ}$ día posoperatorio, aunque la mayoría de los casos se presentan entre el $2^{\circ}$ y el $5^{\circ}$ día. Lo más destacado es la eliminación de grandes volúmenes por la ileostomía de un contenido verde oscuro, habitualmente sin gases, a pesar de lo cual el paciente presenta distensión y dolor abdominal, vómitos y/o la salida de altos débitos por la SNG, lo que en algunos pacientes dura 7 días con un máximo de 40 días en esta serie. Luego del apoyo habitual con la reposición de líquidos y electrolitos, nutrición parenteral y la vigilancia estricta de complicaciones el cuadro revierte en tiempos muy variables sin la realización de acciones específicas o intervenciones mayores. Esta recuperación se mantiene en el tiempo y la mayoría de los pacientes toleran la quimioterapia adyuvante en forma satisfactoria. El tiempo de hospitalización promedio de los pacientes con una DI en nuestra experiencia fue de 21 días, con extremos de 12 y 70 días si se incluyen los reingresos, estadísticamente superior a los pacientes no DI. El impacto de la DI se aprecia en el mayor tiempo de hospitalización y la tasa de reingresos que es significativamente mayor.

Entre las variables propuestas para predecir complicaciones como la HOI y la obstrucción de salida se ha estudiado la relación neutrófilos/linfocitos preoperatoria, que se considera un marcador de actividad inflamatoria ${ }^{13,17}$. En esta serie no hubo diferencias al comparar ambos grupos de pacientes respecto de esta variable, considerando un cut-off de 3.

En conclusión, la DI es una complicación transitoria de gravedad variable que afecta alrededor del 15\% de los pacientes sometidos a una resección radical protegida con una ileostomía en asa y cuya etiología no está clara. La ausencia de dolor de tipo cólico que caracteriza el cuadro sugiere que el mecanismo no es de una obstrucción mecánica, sino más bien una forma de íleo paralítico severo que se manifiesta clínicamente por quiebre de la evolución normal del paciente. Lo más relevante en el examen físico es la marcada distensión abdominal, a pesar de que las pérdidas por la IA y la SNG a veces superan los 8 litros en 24 h. El estudio tomográfico 
Figura 4.

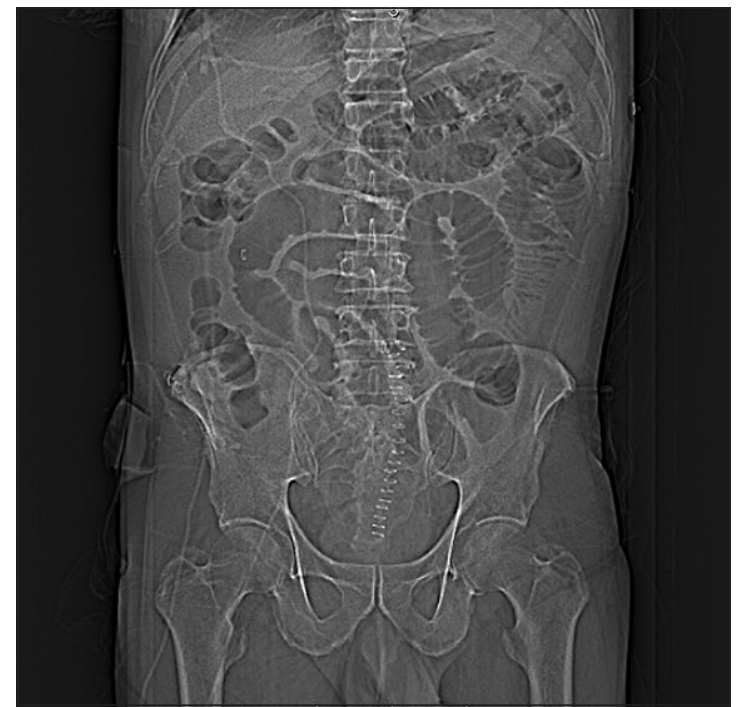

muestra habitualmente una dilatación severa del intestino delgado en la tomografía computada (signo de la pila de monedas con edema de las asas intestinales, Figura 4) y permite descartar una complicación asociada con la cirugía principal. Es llamativo que el estado general del paciente está relativamente conservado y, finalmente, el cuadro revierte en plazos variables con el aporte de volumen vigoroso y, en los casos más graves, requiere de apoyo con nutrición parenteral. No fue posible identificar un perfil del paciente en riesgo de sufrir esta complicación ni precisar factores predictivos. Esta complicación la hemos observado en algunos pacientes intervenidos por enfermedad inflamatoria intestinal con características similares a las descritas.

Las limitaciones de este estudio son claras y evidentes. Es un estudio retrospectivo en un periodo prolongado, el número de casos es escaso $\mathrm{y}$, como se desprende del reporte, no se logró identificar un perfil de paciente en riesgo de una complicación que no es bien comprendida; esta revisión solo pudo caracterizarla con mayor detalle. Con el propósito de avanzar en la comprensión de esta entidad, hemos definido un protocolo de estudio prospectivo de los pacientes que incorpora algunas mediciones de la relación neutrófilos/linfocitos, magnesemia y PCR pre y posoperatorias seriadas, descartar la presencia del Clostridium difficile y el uso precoz del octeótrido subcutáneo ${ }^{18}$ en el manejo de esta complicación.

\section{Responsabilidades éticas}

Protección de personas y animales. Los autores declaran que para esta investigación no se han realizado experimentos en seres humanos ni en animales.

Confidencialidad de los datos. Los autores declaran que en este artículo no aparecen datos de pacientes.

Conflictos de interés: no hay.

\section{Bibliografía}

1. Bannura G, Barrera A, Melo C, Contreras J, Soto D. Resultados del tratamiento con neoadyuvancia del cáncer de recto localmente avanzado. Rev Chil Cir. 2010;62:607-13

2. Garg PK, Goel A, Sharma S, Chishi N, Gaur MK. Protective Diversion Stoma in Low Anterior Resection for Rectal Cancer: A Meta-Analysis of Randomized Controlled Trials. Visc Med. 2019;35:15660 .

3. Ihnát $P$, Guňková $P$, Peteja $M$, Vávra $P$, Pelikán A, Zonča P. Diverting ileostomy in laparoscopic rectal cancer surgery: high price of protection. Surg Endosc. 2016;30:4809-16.

4. Bannura G, Barrera A, Melo C, Illanes F, Gallardo C, Suazo C. Cecostomía amplia: una alternativa de protección de una anastomosis colorrectal baja en cáncer de recto. Rev Chil Cir. 2014;66:345-50.

5. Charlson M, Pompei P, Ales K, Mackenzie C. A new method for classifying prognostic comorbidity in longitudinal studies: development and validation. J Chronic Dis. 1987;40:373-83.

6. Dindo D, Demartines N, Clavien P. Classification of surgical complications. Ann Surg. 2004;240:205-13.

7. Brooke BN. The management of an ileostomy, including its complications. Lancet 1952;2:102-4.

8. Turnbull RB. Management of the ileostomy. Am J Surg. 1953;86:617-24.

9. Vergara-Fernández O, Trejo-Ávila M, Santes O, Solórzano-Vicuña D, SalgadoNesme N. Predictors of dehydration and acute renal failure in patients with diverting ileostomy creation after colorectal surgery. World J Clin Cases
2019;7:1805-13.

10. Fujino S, Myoshi N, Ohue M, Takahashi Y, Yasui M, Sugimura K, et al. Prediction model and treatment of high-output ileostomy in colorectal surgery. Molecular and Clinical Oncol. 2017;7:468-72.

11. Takeda M, Takahashi H, Haragouchi N, Miyoshi N, Hata T, Yamamoto H, et al. Factors predictive of high-output ileostomy: a retrospective single center comparative study. Surgery Today 2019;49:482-7.

12. Baker ML, Williams RN, Nightingale JM. Causes and management of high-output stoma. Colorectal Dis. 2011;13:191-9.

13. Hiramoto Y, Kawahara H, Matsumoto T, Takeda M, Misawa T, Yanaga K. Preoperative neutrophil-lymphocyte ratio is a predictor of high-output ileostomy after colorectal surgery. Anticancer Research 2019;39:3265-8. 
ARTíCULO ORIGINAL

14. Hara Y, Miura T, Sakamoto Y, Morohashi H, Nagase H, Hakamada K. Organ/ space infection is a common cause of high output stoma and outlet obstruction in diverting ileostomy. BMC Surgery 2020;20:83.

15. Tamura K, Matsuda K, Yokohama S, Iwamoto H, Mizumoto Y, Murakami D, et al. Desfunctioning loop ileostomy for rectal anastomoses: predictors of stoma outlet obstruction. Int J Colorectal Dis. 2019;34:1141-5.

16. Fujii $T$, Morita H, Sutoh T, Yajima R, Tsutsumi S, Asao, et al. Outlet obstruction of temporary loop diverting ileostomy. Hepatogastroenterology 2015;62:602-5.

17. Benlice C, Onder A, Babazade R,
Hrabe J, Stocchi L, Steele S, et al. Is the neutrophil-to-lymphocyte ratio associated with increased morbidity after colorectal surgery. Surg Laparosc Endosc Percutan Tech. 2019;29:36-9.

18. Rowe KM, Schiller LR. Ileostomy diarrhea: pathophysiology and management. Proc Bayl Univ Med Cent. 2020;33:218-26 